\title{
Multiple Positive Solutions of a Nonlinear Four-Point Singular Boundary Value Problem with a $p$-Laplacian Operator on Time Scales
}

\author{
Shihuang Hong \\ Institute of Applied Mathematics and Engineering Computations, Hangzhou Dianzi University, \\ Hangzhou 310018, China \\ Correspondence should be addressed to Shihuang Hong, hongshh@hotmail.com \\ Received 29 September 2008; Accepted 12 February 2009 \\ Recommended by Kanishka Perera \\ We present sufficient conditions for the existence of at least twin or triple positive solutions of a \\ nonlinear four-point singular boundary value problem with a $p$-Laplacian dynamic equation on a \\ time scale. Our results are obtained via some new multiple fixed point theorems. \\ Copyright $(2009$ Shihuang Hong. This is an open access article distributed under the Creative \\ Commons Attribution License, which permits unrestricted use, distribution, and reproduction in \\ any medium, provided the original work is properly cited.
}

\section{Introduction}

Let $\mathbf{T}$ be a closed nonempty subset of $\mathbb{R}$. In the light of some of the current literature, $\mathrm{T}$ is called a time scale or measure chain. The following definitions and preliminary notions, which can be found in [1-3], lay out the terms and notation needed later in the discussion.

We will use the convention that, for each interval $J$ of $\mathbb{R}, J_{\mathrm{T}}=J \cap \mathrm{T}$. For $t<\sup \mathrm{T}$ and $r>\inf \mathbf{T}$, we define the forward jump operator, $\sigma$, and the backward jump operator, $\rho$, respectively, by

$$
\sigma(t)=\inf \{\tau \in \mathbf{T}: \tau>t\} \in \mathbf{T}, \quad \rho(r)=\sup \{\tau \in \mathbf{T}: \tau<r\} \in \mathbf{T},
$$

for all $t, r \in \mathbf{T}$. However, $t$ is said to be right-scattered if $\sigma(t)>t$ and $t$ is said to be right-dense (rd) if $\sigma(t)=t$. Also, $t$ is said to be left-scattered if $\rho(t)<t$ and $t$ is said to be left-dense (ld) if $\rho(t)=t$. We introduce the sets $\mathbf{T}^{k}$ and $\mathbf{T}_{k}$ which are derived from the time scale $\mathbf{T}$ as follows. If $\mathbf{T}$ has a right-scattered minimum $m$, then $\mathbf{T}_{k}=\mathbf{T}-\{m\}$; otherwise set $\mathbf{T}_{k}=\mathbf{T}$. If $\mathbf{T}$ has a left-scattered maximum $M$, then $\mathbf{T}^{k}=\mathbf{T}-\{M\}$; otherwise, set $\mathbf{T}^{k}=\mathbf{T}$. 
A function $f$ is left-dense continuous (ld-c, for short) if $f$ is continuous at each left-dense point in $\mathbf{T}$ and its right-sided limits exist at each right-dense points in $\mathbf{T}$. By $C_{\text {ld }}(T,[0, \infty))$ we mean the set of all left-dense continuous functions from $\mathbf{T}$ to $[0, \infty)$.

For $x: \mathbf{T} \rightarrow \mathbb{R}$ and $t \in \mathbf{T}^{k}$, we define the delta derivative of $x(t), x^{\Delta}(t)$, to be the number (when it exists), with the property that, for each $\varepsilon>0$, there exists a neighborhood $U$ of $t$ such that

$$
\left|x(\sigma(t))-x(s)-x^{\Delta}(t)(\sigma(t)-s)\right| \leq \varepsilon|\sigma(t)-s|
$$

for all $s \in U$. We say $x$ is $\Delta$-differentiable at $t$ if its delta derivative exists at $t$, and we say $x$ is $\Delta$-differentiable on $[0,1]_{\mathrm{T}^{k}}$ if its delta derivative exists at each $t \in[0,1]_{\mathrm{T}^{k}}$.

For $x: \mathbf{T} \rightarrow \mathbb{R}$ and $t \in \mathbf{T}_{k}$, we define the nabla derivative of $x(t), x^{\nabla}(t)$, to be the number (when it exists), with the property that, for each $\varepsilon>0$, there exists a neighborhood $U$ of $t$ such that

$$
\left|x(\rho(t))-x(s)-x^{\nabla}(t)(\rho(t)-s)\right| \leq \varepsilon|\rho(t)-s|
$$

for all $s \in U$.

If $\mathbf{T}=\mathbb{R}$, then $x^{\Delta}(t)=x^{\nabla}(t)=x^{\prime}(t)$. If $\mathbf{T}=\mathbb{Z}$, then $x^{\Delta}(t)=x(t+1)-x(t)$ is the forward difference operator while $x^{\nabla}(t)=x(t)-x(t-1)$ is the backward difference operator.

By $C_{\mathrm{ld}}^{\Delta}\left([0,1]_{\mathrm{T}}\right), C_{\mathrm{ld}}^{\nabla}\left([0,1]_{\mathrm{T}}\right)$, we mean the set of all functions from $[0,1]_{\mathrm{T}}$ to $[0, \infty)$ which are $\Delta$-differentiable on $[0,1]_{\mathrm{T}^{k}}, \nabla$-differentiable on $[0,1]_{\mathrm{T}_{k}}$, respectively, and by $C_{\operatorname{lr}}^{\Delta \nabla}\left([0,1]_{\mathrm{T}^{k} \cap \mathrm{T}_{k}}\right)=: C_{\operatorname{lr}}^{\Delta \nabla}$, we mean the set of all functions from $[0,1]_{\mathrm{T}}$ to $[0, \infty)$ which are $\Delta$-differentiable on $[0,1]_{\mathrm{T}^{k}}$ and their delta derivatives are $\nabla$-differentiable on $[0,1]_{\mathrm{T}_{k}}$.

If $F^{\Delta}(t)=f(t)$, then we define the delta integral by

$$
\int_{a}^{t} f(s) \Delta s=F(t)-F(a) .
$$

If $F^{\nabla}(t)=f(t)$, then we define the nabla integral by

$$
\int_{a}^{t} f(s) \nabla s=F(t)-F(a)
$$

Throughout this paper, we assume $\mathbf{T}$ is closed subset of $\mathbb{R}$ with $0 \in \mathbf{T}_{k}$ and $1 \in \mathbf{T}^{k}$. In the same way as the proof of [4, Theorems 2.1,2.3, and 2.10], it is not difficult to verify the following.

Lemma 1.1. Let $f: \mathbf{T} \rightarrow \mathbb{R}$ and $t \in \mathbf{T}^{k}$ (or $t \in \mathbf{T}_{k}$ ). If $f$ is $\Delta$ (or $\nabla$ )-differentiable at $t$, then $f$ is continuous at $t$. Moreover, the following formulas hold:

(i) $\left(\int_{a}^{t} f(s) \Delta s\right)^{\Delta}=f(t)$,

(ii) $\left(\int_{a}^{t} f(s) \Delta s\right)^{\nabla}=f(\rho(t))$,

(iii) $\left(\int_{a}^{t} f(s) \nabla s\right)^{\Delta}=f(\sigma(t))$,

(iv) $\left(\int_{a}^{t} f(s) \nabla s\right)^{\nabla}=f(t)$. 
The theory of time scales was initiated by Hilger [1] as a means of unifying structure for the study of differential equations in the continuous case and the study of finite difference equations in the discrete case and extending theories from differential and difference equations. The theory of dynamical systems on time scales is undergoing rapid development, see [2-10]. This paper is concerned with the multiplicity of positive solutions for the following nonlinear four-point singular boundary value problem of a $p$-Lapalcian dynamic equation on a time scale

$$
\begin{gathered}
{\left[\phi_{p}\left(u^{\Delta}(t)\right)\right]^{\nabla}+a(t) f(u(t))=0, \quad t \in[0,1]_{\mathrm{T}}} \\
m_{1} \phi_{p}(u(0))-n_{1} \phi_{p}\left(u^{\Delta}(\xi)\right)=0, \quad m_{2} \phi_{p}(u(1))+n_{2} \phi_{p}\left(u^{\Delta}(\eta)\right)=0,
\end{gathered}
$$

where $\phi_{p}(s)$ is $p$-Laplacian operator, that is, $\phi_{p}(s)=|s|^{p-2} s, p>1$ (thus, $\phi_{p}(s)$ is strictly increasing on $[0, \infty)),\left(\phi_{p}\right)^{-1}=\phi_{q}, 1 / p+1 / q=1, m_{1}>0, n_{1} \geq 0, m_{2}>0, n_{2} \geq 0, \xi, \eta \in$ $(0, \rho(1))_{\mathrm{T}}$ is prescribed and $\xi<\eta$. Moreover,

(H1) $f: \mathbb{R}^{+} \rightarrow \mathbb{R}^{+}$is continuous ( $\mathbb{R}^{+}$denotes the nonnegative reals),

$(\mathrm{H} 2) a: \mathbf{T} \rightarrow[0,+\infty)$ is ld-c and $0<\int_{I} a(t) \nabla t<+\infty$ for any closed subinterval $I$ of $[0,1]_{\mathrm{T}}$.

Some authors have studied the existence of multiple positive solutions for the nonlinear second-order three-point boundary value problems on time scales, for instance, Anderson [7] has proved that the problem

$$
u^{\Delta \nabla}(t)+f(t, u(t))=0, \quad u(0)=0, \quad a u(\eta)=u(T)
$$

has at least three positive solutions by employing the fixed point theorem due to Leggett and Williams [11], and He [9] has proved that the following problems have twin positive solutions by employing double fixed point theorem due to Avery and Henderson [12]:

$$
\begin{gathered}
{\left[\phi_{p}\left(u^{\Delta}(t)\right)\right]^{\nabla}+a(t) f(u(t))=0, \quad t \in[0, T]_{\mathrm{T}}} \\
u(0)-B_{0}\left(u^{\Delta}(\eta)\right)=0, \quad u^{\Delta}(T)=0, \quad \text { or } \quad u^{\Delta}(0)=0, \quad u(T)+B_{1}\left(u^{\Delta}(\eta)\right)=0 .
\end{gathered}
$$

Hong [13] has proved that the aforementioned problems have triple positive solutions using fixed point theorem due to Avery and Peterson [14]. In this paper, by using fixed point theorems due to Avery and Henderson [12], Avery and Peterson [14], respectively, we prove that there exist at least twin or triple positive solutions to problems (1.6).

In Section 2, we define an operator whose fixed points are solutions to (1.6) and state two fixed point theorems due to $[12,14]$. We also state and prove some lemmas that will be needed in order to prove our main theorems. In Section 3, we state and prove two theorems for the existence of multiple positive solutions of (1.6). Two examples are given in Section 4.

\section{Preliminaries and Lemmas}

The first part of this section is devoted to collect the main terminology and auxiliary results for discussion of fixed points for operators on cones in Banach spaces, which will be foundational in the proof of our main results. 
Let $(E,\|\cdot\|)$ be a real Banach space. A nonempty convex closed set $P$ contained in $E$ is called a cone if the following two conditions are satisfied:

(1) $x \in P, \lambda \geq 0$ implies $\lambda x \in P$,

(2) $x \in P$ and $-x \in P$ implies $x=0$.

The cone $P$ induces an ordering $\leq$ on $E$ by $x \leq y$ if and only if $y-x \in P$.

An operator $F$ is said to be completely continuous if it is continuous and compact (maps bounded sets into relatively compact sets).

For a given cone $P$ in a real Banach space $E$, the map $X: P \rightarrow[0, \infty)$ is called a nonnegative continuous concave function on cone $P$ provided that $X$ is continuous and

$$
x(t x+(1-t) y) \geq t \chi(x)+(1-t) x(y)
$$

for $x, y \in P$ and $0 \leq t \leq 1$. Dual to this, we call the map $\varphi: P \rightarrow[0, \infty)$ a nonnegative continuous convex function on $P$ provided that $\varphi$ is continuous and

$$
\varphi(t x+(1-t) y) \leq t \varphi(x)+(1-t) \varphi(y)
$$

for $x, y \in P$ and $0 \leq t \leq 1$.

Let $\theta, \gamma, \alpha, \psi$ be nonnegative functions on $P$ and $a, b, c$, and $d$ be positive real numbers. We define the following convex sets:

$$
\begin{gathered}
P(\gamma, d)=\{x \in P: \gamma(x)<d\}, \\
P(\gamma, \alpha, b, d)=\{x \in P: b \leq \alpha(x), \gamma(x) \leq d\}, \\
P(\gamma, \theta, \alpha, b, c, d)=\{x \in P: b \leq \alpha(x), \theta(x) \leq c, \gamma(x) \leq d\},
\end{gathered}
$$

and closed sets

$$
\begin{gathered}
\partial P(\gamma, d)=\{x \in P: \gamma(x)=d\}, \\
R(\gamma, \psi, a, d)=\{x \in P: a \leq \psi(x), \gamma(x) \leq d\} .
\end{gathered}
$$

The following two fixed point theorems due to Avery and Henderson [12], Avery and Peterson [14], respectively, are fundamental for us to establish our main results.

Theorem A (see [12]). Let $P$ be a cone in a real Banach space E. Let $\alpha$ and $\delta$ be increasing, nonnegative and continuous functions on $P$, and $\theta$ be a nonnegative, continuous function on $P$ with $\theta(0)=0$ such that for some constants $h>0$ and $d>0$,

$$
\alpha(x) \leq \theta(x) \leq \delta(x), \quad\|x\| \leq h \alpha(x)
$$

for all $x \in \overline{P(\alpha, d)}$. Suppose there exist constants $a$ and $b$ with $0<a<b<d$ such that

$$
\theta(\varepsilon x) \leq \varepsilon \theta(x) \text { for } 0 \leq \varepsilon \leq 1, x \in \partial P(\theta, b)
$$


and $F: \overline{P(\alpha, d)} \rightarrow P$ is a completely continuous operator such that

(s1) $\alpha(F x)>d$ for all $x \in \partial P(\alpha, d)$,

(s2) $\theta(F x)<b$ for all $x \in \partial P(\theta, b)$,

(s3) $P(\delta, a) \neq \varnothing$ and $\delta(F x)>a$ for all $x \in \partial P(\delta, a)$.

Then $F$ has at least two fixed points $x_{1}$ and $x_{2}$ belonging to $\overline{P(\alpha, d)}$ such that

$$
\begin{aligned}
& a<\delta\left(x_{1}\right) \text { with } \theta\left(x_{1}\right)<b, \\
& b<\theta\left(x_{2}\right) \text { with } \alpha\left(x_{2}\right)<d .
\end{aligned}
$$

Theorem B (see [14]). Let $P$ be a cone in a real Banach space E. Let $\theta$ and $\gamma$ be nonnegative continuous convex functions on $P, \alpha$ a nonnegative continuous concave function on $P$ and $\psi a$ nonnegative continuous function on $P$, moreover, $\psi$ satisfy $\psi(\lambda x) \leq \lambda \psi(x)$ for $0 \leq \lambda \leq 1$ such that, for some positive numbers $h$ and $d$,

$$
\alpha(x) \leq \psi(x), \quad\|x\| \leq h \gamma(x)
$$

for all $x \in \overline{P(\gamma, d)}$. Suppose that $F: \overline{P(\gamma, d)} \rightarrow \overline{P(\gamma, d)}$ is a completely continuous operator and there exist positive real numbers $a, b$, and $c$ with $a<b$ such that the following conditions are satisfied:

(h1) $\{x \in P(\gamma, \theta, \alpha, b, c, d): \alpha(x)>b\} \neq \varnothing$ and

$$
\alpha(F x)>b \quad \text { for } x \in P(\gamma, \theta, \alpha, b, c, d) \text {, }
$$

(h2) $\alpha(F x)>b$ for $x \in P(\gamma, \alpha, b, d)$ with $\theta(F x)>c$,

(h3) $0 \notin R(\gamma, \psi, a, d)$ and $\psi(F x)<a$ for $x \in R(\gamma, \psi, a, d)$ with $\psi(x)=a$.

Then $F$ has at least three fixed points $x_{1}, x_{2}, x_{3} \in \overline{P(\gamma, d)}$ such that

$$
\begin{gathered}
r\left(x_{i}\right) \leq d \quad \text { for } i=1,2,3, \\
b<\alpha\left(x_{1}\right), \\
a<\psi\left(x_{2}\right) \quad \text { with } \alpha\left(x_{2}\right)<b, \\
\psi\left(x_{3}\right)<a .
\end{gathered}
$$

In the second part of this section, some lemmas will be proved. A function $u$ is said to be $\mathbf{T}$-concave on $[0,1]_{\mathrm{T}}$ if $u^{\Delta \nabla}(t) \leq 0$ for $t \in[0,1]_{\mathrm{T}^{k} \cap \mathbf{T}_{k}}$. Let $E=C_{\mathrm{ld}}^{\Delta}\left([0,1]_{\mathrm{T}}, \mathbb{R}\right)$ and

$$
\begin{gathered}
|x|_{0}=\max _{t \in[0,1]_{\mathrm{T}}}|x(t)| \\
\|x\|=\max \left\{\max _{t \in[0,1]_{\mathrm{T}}}|x(t)|, \max _{t \in[0,1]_{\mathrm{T}^{k}}}\left|x^{\Delta}(t)\right|\right\} .
\end{gathered}
$$


For given $\sigma \in[0,1]_{\mathrm{T}}$, let us define a set $P$ by

$$
P=\left\{u \in E: u \text { is } \mathbf{T} \text {-concave and nonnegative valued on }[0,1]_{\mathrm{T}}, u^{\Delta}(\sigma)=0\right\}
$$

Clearly, both $\left(E,|\cdot|_{0}\right)$ and $(E,\|\cdot\|)$ are Banach spaces, $P$ is a nonempty subset and a cone of $E$.

Remark 2.1. By (1.6) and its the boundary conditions, we have $u^{\Delta}(\xi) \geq 0, u^{\Delta}(\eta) \leq 0$, and $u^{\Delta \nabla}(t) \leq 0$ for $t \in[0,1]_{\mathrm{T}^{k} \cap \mathrm{T}_{k}}$. Hence $u^{\Delta}(t)$ is decreasing on $[0,1]_{\mathrm{T}}$. This shows that $\sigma \in[\xi, \eta]_{\mathrm{T}^{\prime}}$ that is, $\xi \leq \sigma \leq \eta$.

Lemma 2.2. Let $\lambda \in(0,1 / 2)$ be a given constant with $\lambda<\xi$ and $1-\lambda>\eta$, then for each $u \in P$ one has

$$
u(t) \geq \lambda \max _{s \in[0,1]}|u(s)|, \quad t \in\left[\varrho_{1}, \varrho_{2}\right]_{\mathrm{T}^{\prime}}
$$

where $\rho_{1}=\min \{t \in \mathbf{T}: \lambda \leq t \leq \xi\}$ and $\varrho_{2}=\max \{t \in \mathbf{T}: \eta \leq t \leq 1-\lambda\}$.

Proof. Since $u$ is a T-concave function, we claim that each point on the chord between $\left(t_{1}, u\left(t_{1}\right)\right)$ and $\left(t_{2}, u\left(t_{2}\right)\right)$ with $t_{1}<t_{2}$ is below the graph of $u(t)$. In fact, if there exists $t_{0} \in\left[t_{1}, t_{2}\right]_{\mathrm{T}}$, let $t_{0}=\lambda_{1} t_{1}+\lambda_{2} t_{2}$ for $0 \leq \lambda_{1}, \lambda_{2} \leq 1$ and $\lambda_{1}+\lambda_{2}=1$. Then by the mean-value theorem on time scales (see [6]), there exist $\tau_{1} \in\left[t_{1}, t_{0}\right), \tau_{2} \in\left[t_{0}, t_{2}\right)$ such that

$$
\begin{aligned}
& u\left(t_{0}\right)-u\left(t_{1}\right) \geq u^{\Delta}\left(\tau_{1}\right)\left(t_{0}-t_{1}\right)=u^{\Delta}\left(\tau_{1}\right) \lambda_{2}\left(t_{2}-t_{1}\right), \\
& u\left(t_{2}\right)-u\left(t_{0}\right) \leq u^{\Delta}\left(\tau_{2}\right)\left(t_{2}-t_{0}\right)=u^{\Delta}\left(\tau_{2}\right) \lambda_{1}\left(t_{2}-t_{1}\right) .
\end{aligned}
$$

Multiplying by $-\lambda_{1}$ and $\lambda_{2}$ the aforementioned first and second equations, respectively, then adding up them, we get

$$
\lambda_{2} u\left(t_{2}\right)+\lambda_{1} u\left(t_{1}\right)-u\left(t_{0}\right) \leq\left[u^{\Delta}\left(\tau_{2}\right)-u^{\Delta}\left(\tau_{1}\right)\right] \lambda_{1} \lambda_{2}\left(t_{2}-t_{1}\right) .
$$

Note that $u^{\Delta}(t)$ is $\nabla$-differentiable, we use the mean-value theorem on time scales on $\left[\tau_{1}, \tau_{2}\right]$ again, there exists $\tau \in\left[\tau_{1}, \tau_{2}\right)$ such that

$$
u^{\Delta}\left(\tau_{2}\right)-u^{\Delta}\left(\tau_{1}\right) \leq u^{\Delta \nabla}(\tau)\left(\tau_{2}-\tau_{1}\right)
$$

Loading this into the aforementioned expression, combining $u^{\Delta \nabla}(t) \leq 0$, we have

$$
\lambda_{2} u\left(t_{2}\right)+\lambda_{1} u\left(t_{1}\right)-u\left(\lambda_{1} t_{1}+\lambda_{2} t_{2}\right) \leq \lambda_{1} \lambda_{2} u^{\Delta \nabla}(\tau)\left(\tau_{2}-\tau_{1}\right)\left(t_{2}-t_{1}\right) \leq 0
$$

This yields our claim. 
If $\rho_{1}=\lambda$ and $\rho_{2}=1-\lambda$, the rest of this proof is similar to [15, Lemma 2.2]. Otherwise, it has at least one of two equations that is not holds, say $\rho_{1} \neq \lambda$, thus $\rho_{1}>\lambda$. This implies $\left[\lambda, Q_{1}\right]_{\mathrm{T}}=\varnothing$. Set

$$
u^{*}(t)= \begin{cases}u\left(\rho_{1}\right), & t \in\left[\lambda, \rho_{1}\right] \\ u(t), & t \in\left(\varrho_{1}, 1-\lambda\right]_{\mathrm{T}} .\end{cases}
$$

Again, similar to [15, Lemma 2.2], we can verify the rest of this proof. The proof is similar in other cases. This proof is completed.

Lemma 2.3. Suppose that conditions (H1), (H2) hold. Then $u(t) \in P \cap C_{\operatorname{lr}}^{\Delta \nabla}$ is a solution of boundary value problems (1.6) if and only if $u(t) \in E$ is a solution of the following integral equation:

$$
u(t)=\left\{\begin{array}{l}
\phi_{q}\left(\frac{n_{1}}{m_{1}} \int_{\xi}^{\sigma} a(s) f(u(s)) \nabla s\right)+\int_{0}^{t} \phi_{q}\left(\int_{s}^{\sigma} a(\tau) f(u(\tau)) \nabla \tau\right) \Delta s, \quad t \in[0, \sigma]_{\mathrm{T}}, \\
\phi_{q}\left(\frac{n_{2}}{m_{2}} \int_{\sigma}^{\eta} a(s) f(u(s)) \nabla s\right)+\int_{t}^{1} \phi_{q}\left(\int_{\sigma}^{s} a(\tau) f(u(\tau)) \nabla \tau\right) \Delta s, \quad t \in[\sigma, 1]_{\mathrm{T}},
\end{array}\right.
$$

where $\sigma$ is given in (2.12).

Proof. Necessity. First, by Cauchy $\nabla$-integrating to the equation of $(1.6)$ on $(\sigma, t)_{\mathrm{T}}$, we have

$$
\phi_{p}\left(u^{\Delta}(t)\right)=\phi_{p}\left(u^{\Delta}(\sigma)\right)-\int_{\sigma}^{t} a(s) f(u(s)) \nabla s .
$$

By the definition of $\phi_{p}$ and (2.12), we have $\phi_{p}\left(u^{\Delta}(\sigma)\right)=0$ and $u^{\Delta}(t)=-\phi_{q}\left(\int_{\sigma}^{t} a(s) f(u(s)) \nabla s\right)$, thus, in virtue of Cauchy $\Delta$-integral from $\sigma$ to $t$ of $u^{\Delta}(t)$, we have

$$
u(t)=u(\sigma)-\int_{\sigma}^{t} \phi_{q}\left(\int_{\sigma}^{s} a(\tau) f(u(\tau)) \nabla \tau\right) \Delta s .
$$

Let $t=\eta$ on (2.20), we have $\phi_{p}\left(u^{\Delta}(\eta)\right)=-\int_{\sigma}^{\eta} a(s) f(u(s)) \nabla s$. The boundary condition of (1.6) yields $\phi_{p}(u(1))=-\left(n_{2} / m_{2}\right) \phi_{p}\left(u^{\Delta}(\eta)\right)$, then

$$
u(1)=\phi_{q}\left(\frac{n_{2}}{m_{2}} \int_{\sigma}^{\eta} a(s) f(u(s)) \nabla s\right) .
$$

From (2.21) with $t=1$, together with (2.22), it follows that

$$
u(\sigma)=\phi_{q}\left(\frac{n_{2}}{m_{2}} \int_{\sigma}^{\eta} a(s) f(u(s)) \nabla s\right)+\int_{\sigma}^{1} \phi_{q}\left(\int_{\sigma}^{s} a(\tau) f(u(\tau)) \nabla \tau\right) \Delta s .
$$


Now (2.21) and (2.23) guarantee that, for any $t \in[\sigma, 1]_{\mathrm{T}}$,

$$
u(t)=\phi_{q}\left(\frac{n_{2}}{m_{2}} \int_{\sigma}^{\eta} a(s) f(u(s)) \nabla s\right)+\int_{t}^{1} \phi_{q}\left(\int_{\sigma}^{s} a(\tau) f(u(\tau)) \nabla \tau\right) \Delta s
$$

Similarly, for $t \in[0, \sigma]_{\mathrm{T}}$, by Cauchy $\nabla$-integrating to $(1.6)$ on $(0, \sigma)_{\mathrm{T}}$, it is possible to get

$$
u(t)=\phi_{q}\left(\frac{n_{1}}{m_{1}} \int_{\xi}^{\sigma} a(s) f(u(s)) \nabla s\right)+\int_{0}^{t} \phi_{q}\left(\int_{s}^{\sigma} a(\tau) f(u(\tau)) \nabla \tau\right) \Delta s .
$$

This implies that (2.19) is true.

Sufficiency. Suppose that (2.19) holds. By $\Delta$-differential of (2.19), we have

$$
u^{\Delta}(t)= \begin{cases}\phi_{q}\left(\int_{t}^{\sigma} a(\tau) f(u(\tau)) \nabla \tau\right) \geq 0, & t \in[0, \sigma]_{\mathrm{T}} \\ -\phi_{q}\left(\int_{\sigma}^{t} a(\tau) f(u(\tau)) \nabla \tau\right) \leq 0, & t \in[\sigma, 1]_{\mathrm{T}}\end{cases}
$$

So, by Lemma 1.1 we have $\left(\phi_{p}\left(u^{\Delta}(t)\right)\right)^{\nabla}+a(t) f(u(t))=0, t \in[0,1]_{\mathrm{T}}$. This shows that the first equation of (1.6) holds. Furthermore, taking $t=0$ and $t=1$, respectively on (2.19) and (2.26), we are able to obtain the boundary value equations of (1.6). This proof is completed.

Now, we define a mapping $F: P \rightarrow E$ by

$$
(F u)(t)= \begin{cases}\phi_{q}\left(\frac{n_{1}}{m_{1}} \int_{\xi}^{\sigma} a(s) f(u(s)) \nabla s\right)+\int_{0}^{t} \phi_{q}\left(\int_{s}^{\sigma} a(\tau) f(u(\tau)) \nabla \tau\right) \Delta s, \quad t \in[0, \sigma]_{\mathrm{T}}, \\ \phi_{q}\left(\frac{n_{2}}{m_{2}} \int_{\sigma}^{\eta} a(s) f(u(s)) \nabla s\right)+\int_{t}^{1} \phi_{q}\left(\int_{\sigma}^{s} a(\tau) f(u(\tau)) \nabla \tau\right) \Delta s, \quad t \in[\sigma, 1]_{\mathrm{T}} .\end{cases}
$$

Lemma 2.4. $F: P \rightarrow P$ is completely continuous.

Proof. Note that

$$
(F(u))^{\Delta}(t)= \begin{cases}\phi_{q}\left(\int_{t}^{\sigma} a(\tau) f(u(\tau)) \nabla \tau\right) \geq 0, & t \in[0, \sigma]_{\mathrm{T}} \\ -\phi_{q}\left(\int_{\sigma}^{t} a(\tau) f(u(\tau)) \nabla \tau\right) \leq 0, & t \in[\sigma, 1]_{\mathrm{T}}\end{cases}
$$

we see that $F u \in C_{\text {ld }}^{\Delta}\left([0, T]_{\mathrm{T}},[0, \infty)\right)$ and $(F(u))^{\Delta}(\sigma)=0$. For any given $u \in$ $P$, set $G(t)=(F(u))^{\Delta}(t)$, then $G$ is a continuous function and $G(t) \geq 0$ for 
$t \in[0, \sigma]_{\mathrm{T}}$ and $G(t) \leq 0$ for $t \in[\sigma, 1]_{\mathrm{T}}$. Note that $\phi_{q}$ is increasing, we get that $G$ is decreasing on $[0,1]_{\mathrm{T}}$. Now if $t \in[0,1]_{\mathrm{T}^{k} \cap \mathbf{T}_{k}}$ is left-scattered, from [4, Theorem 2.3] it follows that

$$
G^{\nabla}(t)=\frac{G(\rho(t))-G(t)}{\rho(t)-t} \leq 0
$$

If $t \in[0,1]_{\mathbf{T}^{k} \cap \mathbf{T}_{k}}$ is left-dense, again, from [4, Theorem 2.3], it follows that

$$
G^{\nabla}(t)=\lim _{s \rightarrow t} \frac{G(t)-G(s)}{t-s} \leq 0
$$

Consequently, $(F u)^{\Delta \nabla}(t)=G^{\nabla}(t) \leq 0$ on $[0,1]_{\mathrm{T}^{k} \cap \mathbf{T}_{k}}$. This implies that $F u$ is T-concave on $[0,1]_{\mathrm{T}}$. Therefore, $F(P) \subset P$.

Suppose $D \subset P$ is a bounded set. Let $C>0$ be such that $\|u\| \leq C$ for $u \in D$. For any $u \in D$, note that $|F u|_{0}=(F u)(\sigma)$, we have

$$
\begin{aligned}
|(F u)|_{0} & =(F u)(\sigma) \\
& =\phi_{q}\left(\frac{n_{1}}{m_{1}} \int_{\xi}^{\sigma} a(s) f(u(s)) \nabla s\right)+\int_{0}^{\sigma} \phi_{q}\left(\int_{s}^{\sigma} a(\tau) f(u(\tau)) \nabla \tau\right) \Delta s \\
& \leq\left[\phi_{q}\left(\frac{n_{1}}{m_{1}} \int_{0}^{\sigma} a(s) \nabla s\right)+\int_{0}^{\sigma} \phi_{q}\left(\int_{s}^{\sigma} a(\tau) \nabla \tau\right) \Delta s\right] \phi_{q}\left(\sup _{u \in D} f(u)\right) .
\end{aligned}
$$

In addition, from (2.28) we have

$$
\left|(F u)^{\Delta}(t)\right|=\phi_{q}\left(\int_{t}^{\sigma} a(\tau) f(u(\tau)) \nabla \tau\right) \leq \phi_{q}\left(\int_{0}^{\sigma} a(r) \nabla r\right) \phi_{q}\left(\sup _{u \in D} f(u)\right)
$$

for all $t \in[0, \sigma]_{\mathrm{T}}$ and

$$
\left|(F u)^{\Delta}(t)\right|=\left|-\phi_{q}\left(\int_{\sigma}^{t} a(\tau) f(u(\tau)) \nabla \tau\right)\right| \leq \phi_{q}\left(\int_{\sigma}^{1} a(r) \nabla r\right) \phi_{q}\left(\sup _{u \in D} f(u)\right)
$$

for all $t \in[\sigma, 1]_{\mathrm{T}}$. This yields that $F(D)$ is bounded in the norm $|\cdot|_{0}$ or $\|\cdot\|$. Furthermore, it is easy to see by the Arzela-Ascoli theorem and Lebesgue dominated convergent theorem that $F$ is completely continuous. The proof is completed.

\section{Main Results}

In this section, we consider the existence of twin or triple positive solutions for (1.6). Let us start by defining that the function $u:[0,1]_{\mathrm{T}} \rightarrow \mathbb{R}$ is called a solution of (1.6) if $u$ is $\Delta$ differentiable, $u^{\Delta}:[0,1]_{\mathrm{T}^{k}} \rightarrow \mathbb{R}$ is nabla differentiable on $[0,1]_{\mathrm{T}^{k} \cap \mathbf{T}_{k}}, u^{\Delta \nabla}:[0,1]_{\mathrm{T}^{k} \cap \mathbf{T}_{k}} \rightarrow \mathbb{R}$ is continuous, and $u$ satisfies the boundary value problem (1.6). 
We first deal with the existence of double positive solutions of (1.6). Let $\lambda, \rho_{1}$ and $\rho_{2}$ be as in Lemma 2.2 and define the increasing, nonnegative, and continuous functions $\gamma, \theta, \alpha$ on $P$ by

$$
\begin{gathered}
\theta(x)=\max _{t \in\left[0, \rho_{1}\right]_{\mathrm{T}}}|x(t)|, \quad \alpha(x)=\min _{t \in\left[\rho_{1}, \rho_{2}\right]_{\mathrm{T}}} x(t), \\
\delta(x)=\min _{t \in\left[\rho_{1}, \sigma\right]_{\mathrm{T}}} x(t) .
\end{gathered}
$$

Remark 3.1. From the fact that $x$ is $\mathbf{T}$-concave on $[0,1]_{\mathrm{T}}$, we see that $x^{\Delta}(t)$ is decreasing on $[0,1]_{\mathrm{T}^{k}}$. Consequently, we obtain that $x^{\Delta}(t) \geq x^{\Delta}(\sigma)=0$ for $t \in[0, \sigma]_{\mathrm{T}^{k}}$ and $x^{\Delta}(t) \leq x^{\Delta}(\sigma)=0$ for $t \in[\sigma, 1]_{\mathrm{T}^{k}}$. This implies that $x(t)$ is increasing on $[0, \sigma]_{\mathrm{T}}$ and decreasing on $[\sigma, 1]_{\mathrm{T}}$. Hence,

$$
\theta(x)=x\left(\varrho_{1}\right)=\delta(x), \quad \alpha(x)=\min \left\{x\left(\varrho_{1}\right), x\left(\varrho_{2}\right)\right\} .
$$

For notational convenience, we denote $\mathcal{S}_{1}, \mathcal{S}_{2}$, and $M$ by

$$
\begin{aligned}
& \mathcal{S}_{1}=\min _{t \in[\xi, \eta]_{\mathrm{T}}}\left[\phi_{q}\left(\frac{n_{1}}{m_{1}} \int_{\xi}^{t} a(s) \nabla s\right)+\lambda \phi_{q}\left(\int_{Q_{1}}^{t} a(\tau) \nabla \tau\right)\right], \\
& \mathcal{S}_{2}=\min _{t \in[\xi, \eta]_{\mathrm{T}}}\left[\phi_{q}\left(\frac{n_{2}}{m_{2}} \int_{t}^{\eta} a(s) \nabla s\right)+\lambda \phi_{q}\left(\int_{t}^{\rho_{2}} a(\tau) \nabla \tau\right)\right], \\
& M=\phi_{q}\left(\frac{n_{1}}{m_{1}} \int_{\xi}^{\eta} a(s) \nabla s\right)+\rho_{1} \phi_{q}\left(\int_{0}^{\eta} a(\tau) \nabla \tau\right) .
\end{aligned}
$$

In addition, let us impose the following hypotheses on $f$ :

(D1) $f(w)>\phi_{p}(d / \mathcal{S})$ for $\mathcal{S}=\min \left\{\mathcal{S}_{1}, \mathcal{S}_{2}\right\}$ and $w \in[d,(1 / \lambda) d]$;

(D2) $f(w)<\phi_{p}(b / M)$ for $w \in[0,(1 / \lambda) b]$;

(D3) $f(w)>\phi_{p}\left(a / \mathcal{S}_{1}\right)$ for $w \in[a,(1 / \lambda) a]$.

Here constants $a, b, d$ satisfy $0<a<b<\lambda d$ and $a / \mathcal{S}_{1}<b / M$. In the following theorem, we will work in the Banach space $\left(E,|\cdot|_{0}\right)$.

Theorem 3.2. Assume that conditions (H1)-(H2) and (D1)-(D3) hold, then (1.6) has at least two positive solutions $x_{1}, x_{2}$ such that

$$
\begin{aligned}
& a<\min _{t \in\left[\rho_{1}, \sigma\right]_{\mathrm{T}}} x_{1}(t) \quad \text { with } \max _{t \in\left[0, \rho_{1}\right]_{\mathrm{T}}} x_{1}(t)<b, \\
& b<\max _{t \in\left[0, \rho_{1}\right]_{\mathrm{T}}} x_{2}(t) \quad \text { with } \min _{t \in\left[\rho_{1}, \rho_{2}\right]_{\mathrm{T}}} x_{2}(t)<d .
\end{aligned}
$$

Proof. To obtain the result of Theorem 3.2, it is sufficient from Lemma 2.3 to show that $F$ has at least two fixed points. To this purpose, we show that all conditions of Theorem A are fulfilled. We see that, for each $x \in P$,

$$
\alpha(x) \leq x\left(\rho_{1}\right)=\theta(x)=\delta(x),
$$


and from Lemma 2.2 it follows that

$$
|x|_{0} \leq \frac{1}{\lambda} \min \left\{x\left(\rho_{1}\right), x\left(\rho_{2}\right)\right\}=\frac{1}{\lambda} \alpha(x) .
$$

Obviously, $\theta(\varepsilon x)=\varepsilon \theta(x)$ for $\varepsilon \in[0,1]$ and $x \in \partial P(\theta, b)$. Lemma 2.4 guarantees that $F$ maps $P(\alpha, d)$ into $P$. Now we verify that condition (s1) holds. Choose $x \in \partial P(\alpha, d)$, that is, $\alpha(x)=$ $\min _{t \in\left[\rho_{1}, \rho_{2}\right]_{\mathrm{T}}} x(t)=\min \left\{x\left(\rho_{1}\right), x\left(\rho_{2}\right)\right\}=d$. This implies that $x(t) \geq d$ for all $t \in\left[\rho_{1}, \varrho_{2}\right]_{\mathrm{T}}$. From Lemma 2.2, we have

$$
d \leq x(t) \leq \frac{1}{\lambda} d, \quad t \in\left[\varrho_{1}, \varrho_{2}\right]_{\mathrm{T}} .
$$

As a consequence of (D1),

$$
f(x(t))>\phi_{p}\left(\frac{d}{\mathcal{S}}\right), \quad t \in\left[\varrho_{1}, \varrho_{2}\right]_{\mathrm{T}} .
$$

Since $F x \in P$, we have

$$
\begin{aligned}
&(F x)\left(\rho_{1}\right)=\phi_{q}\left(\frac{n_{1}}{m_{1}} \int_{\xi}^{\sigma} a(s) f(x(s)) \nabla s\right)+\int_{0}^{\rho_{1}} \phi_{q}\left(\int_{s}^{\sigma} a(\tau) f(x(\tau)) \nabla \tau\right) \Delta s \\
& \geq \phi_{q}\left(\frac{n_{1}}{m_{1}} \int_{\xi}^{\sigma} a(s) f(x(s)) \nabla s\right)+\int_{0}^{\rho_{1}} \phi_{q}\left(\int_{\rho_{1}}^{\sigma} a(\tau) f(x(\tau)) \nabla \tau\right) \Delta s \\
&>\left[\phi_{q}\left(\frac{n_{1}}{m_{1}} \int_{\xi}^{\sigma} a(s) \nabla s\right)+\rho_{1} \phi_{q}\left(\int_{\rho_{1}}^{\sigma} a(\tau) \nabla \tau\right)\right] \frac{d}{\mathcal{S}} \\
& \geq\left[\phi_{q}\left(\frac{n_{1}}{m_{1}} \int_{\xi}^{\sigma} a(s) \nabla s\right)+\lambda \phi_{q}\left(\int_{\rho_{1}}^{\sigma} a(\tau) \nabla \tau\right)\right] \frac{d}{\mathcal{S}} \\
& \geq d, \\
&(F x)\left(\rho_{2}\right)=\phi_{q}\left(\frac{n_{2}}{m_{2}} \int_{\sigma}^{\eta} a(s) f(x(s)) \nabla s\right)+\int_{Q_{2}}^{1} \phi_{q}\left(\int_{\sigma}^{s} a(\tau) f(x(\tau)) \nabla \tau\right) \Delta s \\
& \geq \phi_{q}\left(\frac{n_{2}}{m_{2}} \int_{\sigma}^{\eta} a(s) f(x(s)) \nabla s\right)+\int_{Q_{2}}^{1} \phi_{q}\left(\int_{\sigma}^{\rho_{2}} a(\tau) f(x(\tau)) \nabla \tau\right) \Delta s \\
&> {\left[\phi_{q}\left(\frac{n_{2}}{m_{2}} \int_{\sigma}^{\eta} a(s) \nabla s\right)+\left(1-\rho_{2}\right) \phi_{q}\left(\int_{\sigma}^{\rho_{2}} a(\tau) \nabla \tau\right)\right] \frac{d}{\mathcal{S}} } \\
& \geq {\left[\phi_{q}\left(\frac{n_{2}}{m_{2}} \int_{\sigma}^{\eta} a(s) \nabla s\right)+\lambda \phi_{q}\left(\int_{\sigma}^{\rho_{2}} a(\tau) \nabla \tau\right)\right] \frac{d}{\mathcal{S}} } \\
& \geq d .
\end{aligned}
$$

This implies that $\alpha(x)>d$, for all $x \in \partial P(\alpha, d)$, that is, (s1) is true. 
Next, we cheek the condition (s2). For $x \in \partial P(\theta, b)$, we have $\theta(x)=x\left(\rho_{1}\right)=b$. Lemma 2.2 shows that $\max _{t \in[0,1]_{\mathrm{T}}} x(t) \leq(1 / \lambda) x\left(\rho_{1}\right)=(1 / \lambda) b$. Therefore,

$$
0 \leq x(t) \leq \frac{1}{\lambda} b, \quad t \in[0,1]_{\mathrm{T}} .
$$

(D2) guarantees $f(x(t))<\phi_{p}(b / M)$ for $t \in[0,1]_{\mathrm{T}}$. Thus,

$$
\begin{aligned}
\theta(F x) & =(F x)\left(\rho_{1}\right) \\
& =\phi_{q}\left(\frac{n_{1}}{m_{1}} \int_{\xi}^{\sigma} a(s) f(x(s)) \nabla s\right)+\int_{0}^{\rho_{1}} \phi_{q}\left(\int_{s}^{\sigma} a(\tau) f(x(\tau)) \nabla \tau\right) \Delta s \\
& \leq \phi_{q}\left(\frac{n_{1}}{m_{1}} \int_{\xi}^{\eta} a(s) f(x(s)) \nabla s\right)+\int_{0}^{\rho_{1}} \phi_{q}\left(\int_{0}^{\eta} a(\tau) f(x(\tau)) \nabla \tau\right) \Delta s \\
& <\left[\phi_{q}\left(\frac{n_{1}}{m_{1}} \int_{\xi}^{\eta} a(s) \nabla s\right)+\rho_{1} \phi_{q}\left(\int_{0}^{\eta} a(\tau) \nabla \tau\right)\right] \frac{b}{M} \\
& =b .
\end{aligned}
$$

This implies that (s2) holds.

Finally, we verify that the condition (s3) is satisfied. Take $x(t)=a / 2$, for all $t \in$ $[0,1]_{\mathrm{T}}$, then $\delta(x)=a / 2<a$. This yields that $P(\delta, a) \neq \varnothing$. Let $x \in \partial P(\delta, a)$, then $\delta(x)=$ $\min _{t \in\left[\rho_{1}, \sigma\right]_{\mathrm{T}}} x(t)=x\left(\rho_{1}\right)=a$. Again, Lemma 2.2 guarantees that

$$
a \leq x(t) \leq \frac{1}{\lambda} a, \quad t \in\left[\rho_{1}, \sigma\right]_{\mathrm{T}}
$$

From (D3), we have $f(x(t))>\phi_{p}\left(a / \mathcal{S}_{1}\right)$ for $t \in\left[\rho_{1}, \sigma\right]_{\mathrm{T}}$. Hence, we have

$$
\begin{aligned}
\delta(F x) & =(F x)\left(\rho_{1}\right) \\
& =\phi_{q}\left(\frac{n_{1}}{m_{1}} \int_{\xi}^{\sigma} a(s) f(x(s)) \nabla s\right)+\int_{0}^{\rho_{1}} \phi_{q}\left(\int_{s}^{\sigma} a(\tau) f(x(\tau)) \nabla \tau\right) \Delta s \\
& \geq \phi_{q}\left(\frac{n_{1}}{m_{1}} \int_{\xi}^{\sigma} a(s) f(x(s)) \nabla s\right)+\int_{0}^{\rho_{1}} \phi_{q}\left(\int_{\rho_{1}}^{\sigma} a(\tau) f(x(\tau)) \nabla \tau\right) \Delta s \\
& >\left[\phi_{q}\left(\frac{n_{1}}{m_{1}} \int_{\xi}^{\sigma} a(s) \nabla s\right)+\lambda \phi_{q}\left(\int_{\rho_{1}}^{\sigma} a(\tau) \nabla \tau\right)\right] \frac{a}{\mathcal{S}_{1}} \\
& \geq a .
\end{aligned}
$$

Consequently, Theorem A implies that $F$ has at least two fixed points which are positive solutions and satisfy Theorem 3.2. This proof is completed. 
In what follows, we discuss the existence of three positive solutions of (1.6) and we will work in the Banach space $(E,\|\cdot\|)$. We need the positive numbers $\mathcal{S}_{1}, \mathcal{S}_{2}$ and $\mathcal{K}, \boldsymbol{M}$ defined by

$$
\begin{aligned}
& \mathcal{K}=\max \left\{\phi_{q}\left(\int_{0}^{\eta} a(s) \nabla s\right), \phi_{q}\left(\int_{\xi}^{1} a(s) \nabla s\right)\right\}, \\
& \mathcal{M}=\phi_{q}\left(\frac{n_{1}}{m_{1}} \int_{\xi}^{\eta} a(s) \nabla s\right)+\eta \phi_{q}\left(\int_{0}^{\eta} a(\tau) \nabla \tau\right) .
\end{aligned}
$$

We will consider the problem (1.6) under the following assumptions:

(C1) $f(w) \leq \phi_{p}(d / N)$ with $N=\max \{\mathcal{M}, \mathcal{K}\}$ and $w \in[0, d]$;

(C2) $f(w)>\phi_{p}(b / \mathcal{S})$ with $\mathcal{S}=\min \left\{\mathcal{S}_{1}, \mathcal{S}_{2}\right\}$ and $w \in[b,(1 / \lambda) b]$;

(C3) $f(w)<\phi_{p}(a / \mathcal{M})$ for $w \in[0, \lambda a]$.

Here constants $a, b, d$ satisfy $0<a<b<(\lambda / 2) d$ and $b / \mathcal{S} \leq d / N$.

For $x \in P$, define

$$
\begin{gathered}
r(x)=\|x\|, \quad \theta(x)=\max _{t \in[0,1]_{\mathrm{T}}}|x(t)| \\
\alpha(x)=\min _{t \in\left[\rho_{1}, \rho_{2}\right]_{\mathrm{T}}} x(t), \quad \psi(x)=\max _{t \in\left[\rho_{1}, \rho_{2}\right]_{\mathrm{T}}} x(t),
\end{gathered}
$$

where $\lambda, \rho_{1}$ and $\rho_{2}$ are given as in Lemma 2.2.

Remark 3.3. Clearly, $\gamma$ and $\theta$ are nonnegative continuous convex functions, $\alpha$ is the nonnegative continuous concave function and $\psi$ is nonnegative continuous function on the cone $P$. In view of Remark 3.1, we see clearly that $\alpha(x) \leq \psi(x)=x(\sigma)$. Hence, condition (2.8) is satisfied with $h=1$. We also have that $\psi(\pi x)=\pi \psi(x)$ for $\pi \in[0,1]$ and $x \in P$. Remark 3.1 shows that $\alpha(x)=\min \left\{x\left(\rho_{1}\right), x\left(\rho_{2}\right)\right\}$.

Theorem 3.4. If the conditions (H1)-(H2) and (C1)-(C3) hold, then (1.6) has at least three positive solutions $x_{1}, x_{2}$, and $x_{3}$ satisfying

$$
\begin{gathered}
\left\|x_{i}\right\| \leq d \text { for } i=1,2,3 ; \\
b<\min \left\{\left|x_{1}(t)\right|: t \in\left[\varrho_{1}, \varrho_{2}\right]_{\mathrm{T}}\right\} ; \\
a<\max \left\{\left|x_{2}(t)\right|: t \in\left[\varrho_{1}, \varrho_{2}\right]_{\mathrm{T}}\right\} \text { with } \min \left\{\left|x_{2}(t)\right|: t \in\left[\varrho_{1}, \varrho_{2}\right]_{\mathrm{T}}\right\}<b ; \\
\max \left\{\left|x_{3}(t)\right|: t \in[0,1]_{\mathrm{T}}\right\}<a .
\end{gathered}
$$

Proof. To obtain the result of Theorem 3.4, it is sufficient from Lemma 2.3 to show that $F$ has at least three fixed points. To this purpose, We show that all conditions of Theorem B are fulfilled. we will divide this proof into three steps. 
Step 1. We will prove that $F$ maps $\overline{P(\gamma, d)}$ into itself. In fact, for each $u \in \overline{P(\gamma, d)}$, from $\gamma(u)=$ $\|u\| \leq d$ and $(\mathrm{C} 1)$, it follows that

$$
f(u(r)) \leq \phi_{p}\left(\frac{d}{N}\right) \text { for } r \in[0,1]_{\mathrm{T}} .
$$

Applying this, together with $F u \in P$, we have the following estimate:

$$
\begin{aligned}
\max _{t \in[0,1]_{\mathrm{T}}}|(F u)(t)| & =(F u)(\sigma) \\
& =\phi_{q}\left(\frac{n_{1}}{m_{1}} \int_{\xi}^{\sigma} a(s) f(u(s)) \nabla s\right)+\int_{0}^{\sigma} \phi_{q}\left(\int_{s}^{\sigma} a(\tau) f(u(\tau)) \nabla \tau\right) \Delta s \\
& \leq \phi_{q}\left(\frac{n_{1}}{m_{1}} \int_{\xi}^{\sigma} a(s) f(u(s)) \nabla s\right)+\int_{0}^{\sigma} \phi_{q}\left(\int_{0}^{\sigma} a(\tau) f(u(\tau)) \nabla \tau\right) \Delta s \\
& \leq\left[\phi_{q}\left(\frac{n_{1}}{m_{1}} \int_{\xi}^{\eta} a(s) \nabla s\right)+\eta \phi_{q}\left(\int_{0}^{\eta} a(\tau) \nabla \tau\right)\right] \frac{d}{N} \\
& \leq d .
\end{aligned}
$$

From (2.28), we also have

$$
\left|(F u)^{\Delta}(t)\right|=\phi_{q}\left(\int_{t}^{\sigma} a(\tau) f(u(\tau)) \nabla \tau\right) \leq \phi_{q}\left(\int_{0}^{\eta} a(r) \nabla r\right) \frac{d}{N} \leq d,
$$

for all $t \in[0, \sigma]_{\mathrm{T}}$ and

$$
\left|(F u)^{\Delta}(t)\right|=\left|-\phi_{q}\left(\int_{\sigma}^{t} a(\tau) f(u(\tau)) \nabla \tau\right)\right| \leq \phi_{q}\left(\int_{\xi}^{1} a(r) \nabla r\right) \frac{d}{N} \leq d,
$$

for all $t \in[\sigma, 1]_{\mathrm{T}}$. Hence, $\gamma(F u)=\|F u\| \leq d$. By Lemma 2.4, we deduce that $F$ maps $\overline{P(\gamma, d)}$ into itself.

Step 2. To check condition (h1), we choose $u(t)=(1 / \lambda) b, t \in[0,1]_{\mathrm{T}}$. It is easy to see that $u(t)=$ $(1 / \lambda) b \in P(\gamma, \theta, \alpha, b,(1 / \lambda) b, d)$ and $\alpha(u)=(1 / \lambda) b>b$, that is, $\{u \in P(\gamma, \theta, \alpha, b,(1 / \lambda) b, d)$ : $\alpha(u)>b\} \neq \varnothing$. For any $u \in P(\gamma, \theta, \alpha, b,(1 / \lambda) b, d)$, then $b \leq u(t) \leq(1 / \lambda) b$ for all $t \in\left[\rho_{1}, \varrho_{2}\right]_{\mathrm{T}}$. Assumption (C2) guarantees

$$
f(u(r))>\phi_{p}\left(\frac{b}{\mathcal{S}}\right) \quad \forall r \in\left[\varrho_{1}, \varrho_{2}\right]_{\mathrm{T}}
$$


Since $F u \in P$, we have

$$
\begin{aligned}
(F u)\left(\rho_{1}\right) & =\phi_{q}\left(\frac{n_{1}}{m_{1}} \int_{\xi}^{\sigma} a(s) f(u(s)) \nabla s\right)+\int_{0}^{\rho_{1}} \phi_{q}\left(\int_{s}^{\sigma} a(\tau) f(u(\tau)) \nabla \tau\right) \Delta s \\
& \geq \phi_{q}\left(\frac{n_{1}}{m_{1}} \int_{\xi}^{\sigma} a(s) f(u(s)) \nabla s\right)+\int_{0}^{\rho_{1}} \phi_{q}\left(\int_{\rho_{1}}^{\sigma} a(\tau) f(u(\tau)) \nabla \tau\right) \Delta s \\
& =\left[\phi_{q}\left(\frac{n_{1}}{m_{1}} \int_{\xi}^{\sigma} a(s) \nabla s\right)+\rho_{1} \phi_{q}\left(\int_{\rho_{1}}^{\sigma} a(\tau) \nabla \tau\right)\right] \frac{d}{\mathcal{S}} \\
& \geq\left[\phi_{q}\left(\frac{n_{1}}{m_{1}} \int_{\xi}^{\sigma} a(s) \nabla s\right)+\lambda \phi_{q}\left(\int_{\rho_{1}}^{\sigma} a(\tau) \nabla \tau\right)\right] \frac{b}{\mathcal{S}} \\
& \geq b, \\
(F u)\left(\rho_{2}\right) & =\phi_{q}\left(\frac{n_{2}}{m_{2}} \int_{\sigma}^{\eta} a(s) f(u(s)) \nabla s\right)+\int_{Q_{2}}^{1} \phi_{q}\left(\int_{\sigma}^{s} a(\tau) f(u(\tau)) \nabla \tau\right) \Delta s \\
& \geq \phi_{q}\left(\frac{n_{2}}{m_{2}} \int_{\sigma}^{\eta} a(s) f(u(s)) \nabla s\right)+\int_{Q_{2}}^{1} \phi_{q}\left(\int_{\sigma}^{\rho_{2}} a(\tau) f(u(\tau)) \nabla \tau\right) \Delta s \\
& >\left[\phi_{q}\left(\frac{n_{2}}{m_{2}} \int_{\sigma}^{\eta} a(s) \nabla s\right)+\left(1-\rho_{2}\right) \phi_{q}\left(\int_{\sigma}^{\rho_{2}} a(\tau) \nabla \tau\right)\right] \frac{d}{\mathcal{S}} \\
& \geq\left[\phi_{q}\left(\frac{n_{2}}{m_{2}} \int_{\sigma}^{\eta} a(s) \nabla s\right)+\lambda \phi_{q}\left(\int_{\sigma}^{\rho_{2}} a(\tau) \nabla \tau\right)\right] \frac{b}{\mathcal{S}} \\
& b .
\end{aligned}
$$

By Remark 3.3, we infer that $\alpha(F(u))>b$ for all $u \in P(\gamma, \theta, \alpha, b,(1 / \lambda) b, d)$. This shows that condition (h1) is true.

Step 3. It remains to prove (in virtue of Theorem B) that conditions (h2) and (h3) hold. have

We first check (h2). For any $u \in P(\gamma, \alpha, b, d)$ with $\theta(F u)>(1 / \lambda) b$, from Lemma 2.2, we

$$
\begin{aligned}
& (F u)\left(\rho_{1}\right) \geq \lambda \theta(F u)>\lambda \frac{1}{\lambda} b=b, \\
& (F u)\left(\rho_{2}\right) \geq \lambda \theta(F u)>\lambda \frac{1}{\lambda} b=b .
\end{aligned}
$$

So, by means of Remark 3.3, we have $\alpha(F u)>b$. This implies that (h2) is true.

Finally, we check condition (h3). Clearly, as $\psi(0)=0<a$, we have $0 \notin R(\gamma, \psi, a, d)$. Suppose that $u \in R(\gamma, \psi, a, d)$ with $\psi(u)=\max _{t \in[0,1]_{\mathrm{T}}} u(t)=u(\sigma)=a$. Then, in virtue of 
Lemma 2.2, we have

$$
\max _{t \in[0,1]_{\mathrm{T}}}|u(t)| \leq \lambda u(\sigma)=\lambda a
$$

This yields (note that $u$ is nonnegative)

$$
0 \leq u(t) \leq \lambda a \text { for } t \in[0,1]_{\mathrm{T}} .
$$

By assumption (C3), we have

$$
f(u(r))<\phi_{p}\left(\frac{a}{\mathcal{M}}\right) \text { for } r \in[0,1]_{\mathrm{T}} .
$$

Thereby

$$
\begin{aligned}
\psi(F u) & =(F u)(\sigma) \\
& =\phi_{q}\left(\frac{n_{1}}{m_{1}} \int_{\xi}^{\sigma} a(s) f(u(s)) \nabla s\right)+\int_{0}^{\sigma} \phi_{q}\left(\int_{s}^{\sigma} a(\tau) f(u(\tau)) \nabla \tau\right) \Delta s \\
& \leq \phi_{q}\left(\frac{n_{1}}{m_{1}} \int_{\xi}^{\sigma} a(s) f(u(s)) \nabla s\right)+\int_{0}^{\sigma} \phi_{q}\left(\int_{0}^{\sigma} a(\tau) f(u(\tau)) \nabla \tau\right) \Delta s \\
& <\left[\phi_{q}\left(\frac{n_{1}}{m_{1}} \int_{\xi}^{\eta} a(s) \nabla s\right)+\eta \phi_{q}\left(\int_{0}^{\eta} a(\tau) \nabla \tau\right)\right] \frac{a}{\mathcal{M}} \\
& =a,
\end{aligned}
$$

which implies $\psi(F u)<a$. So, condition (h3) holds.

Conclusively, we obtain that (1.6) has at least three solutions $x_{1}, x_{2}$, and $x_{3}$ satisfying Theorem 3.4. The proof is completed.

\section{Two Examples}

Example 4.1. Let $\mathbf{T}=\left\{1-(1 / 2)^{z}: z \in \mathbb{N}\right\} \cup\{1 / 16,1 / 8,1 / 4,1\}$ (N stands for the natural number set). If function $f: \mathbb{R} \rightarrow \mathbb{R}^{+}$is defined by

$$
f(x)= \begin{cases}1+x^{2}, & x \leq \frac{\sqrt{2}-1}{8}, \\ 1+\left(x-\frac{\sqrt{2}+7}{8}\right)^{2}, & \frac{\sqrt{2}-1}{8}<x \leq 8, \\ 1+\left(x-\frac{\sqrt{2}+7}{8}\right)^{2}+2782(x-8), & x>8,\end{cases}
$$


then the condition (H1) holds. Taking $\lambda=1 / 16, \xi=1 / 8, \sigma=1 / 4, \eta=1 / 2, p=2, m_{1}=n_{1}=$ $m_{2}=n_{2}=1$, and $A(t)=(1 / 2) \sqrt{t}$, then

$$
a(t)=A^{\nabla}(t)= \begin{cases}\frac{1}{2(\sqrt{\rho(t)}+\sqrt{t})}, & \rho(t)<t \\ \frac{1}{4 \sqrt{t}}, & \rho(t)=t\end{cases}
$$

satisfying condition (H2). Clearly,

$$
\begin{aligned}
\mathcal{S}_{1} & =\min _{t \in[1 / 8,1 / 2]}\left[\phi_{q}\left(\int_{1 / 8}^{t} a(s) \nabla s\right)+\frac{1}{16} \phi_{q}\left(\int_{1 / 16}^{t} a(\tau) \nabla \tau\right)\right] \\
& =\frac{1}{64 \sqrt{2}}-\frac{1}{128}, \\
\mathcal{S}_{2} & =\min _{t \in[1 / 8,1 / 2]}\left[\phi_{q}\left(\int_{t}^{1 / 2} a(s) \nabla s\right)+\frac{1}{16} \phi_{q}\left(\int_{t}^{15 / 16} a(\tau) \nabla \tau\right)\right] \\
& =\frac{\sqrt{15}}{128}-\frac{1}{32 \sqrt{2}}, \\
M & =\phi_{q}\left(\int_{1 / 8}^{1 / 2} a(s) \nabla s\right)+\frac{1}{16} \phi_{q}\left(\int_{0}^{1 / 2} a(s) \nabla s\right) \\
& =\frac{9}{32 \sqrt{2}} .
\end{aligned}
$$

Now we choose $a=(\sqrt{2}-1) / 128, b=1 / 2, d=9$, then $f(x)$ satisfies

$$
\begin{gathered}
f(x)>\phi_{p}\left(\frac{d}{\mathcal{S}}\right)=\frac{1152}{\sqrt{2}-1}, \quad x \in[9,144] \\
f(x)<\phi_{p}\left(\frac{b}{M}\right)=\frac{16 \sqrt{2}}{9}, \quad x \in[0,8] \\
f(x)>\phi_{p}\left(\frac{a}{\mathcal{S}_{1}}\right)=1, \quad x \in\left[\frac{\sqrt{2}-1}{128}, \frac{\sqrt{2}-1}{8}\right] .
\end{gathered}
$$

Consequently, all assumptions of Theorem 3.2 hold. Hence, by Theorem 3.2, the boundary value problems (1.6) has at least two positive solutions $x_{1}$ and $x_{2}$ satisfying

$$
\begin{aligned}
& \frac{\sqrt{2}-1}{128}<\min _{t \in[1 / 16,1 / 4]_{\mathrm{T}}} x_{1}(t) \quad \text { with } \max _{t \in[0,1 / 16]_{\mathrm{T}}} x_{1}(t)<\frac{1}{2} \\
& \frac{1}{2}<\max _{t \in[0,1 / 16]_{\mathrm{T}}} x_{2}(t) \quad \text { with } \min _{t \in[1 / 16,15 / 16]_{\mathrm{T}}} x_{2}(t)<9 .
\end{aligned}
$$


Example 4.2. Let the set $\mathbf{T}$, the functions $a$, and all parameters be the same as those in Example 4.1. Then we have

$$
\begin{gathered}
\mathcal{K}=\max \left\{\phi_{q}\left(\int_{0}^{1 / 2} a(s) \nabla s\right), \phi_{q}\left(\int_{1 / 8}^{1} a(s) \nabla s\right)\right\}=\frac{\sqrt{2}}{4} \\
\mathcal{M}=\phi_{q}\left(\int_{1 / 8}^{1 / 2} a(s) \nabla s\right)+\frac{1}{2} \phi_{q}\left(\int_{0}^{1 / 2} a(\tau) \nabla \tau\right)=\frac{\sqrt{2}}{4} .
\end{gathered}
$$

Consider the function

$$
f(x)= \begin{cases}\sqrt{x}, & 0 \leq x \leq \frac{1}{16} \\ \frac{1}{4}+\frac{256(16 x-1)^{2}}{961(\sqrt{2}-1)}, & \frac{1}{16}<x \leq 2 \\ \frac{(x-2)^{2}}{95000}+\frac{256}{\sqrt{2}-1}+\frac{1}{4}, & x>2 .\end{cases}
$$

Let us choose $a=1, b=2, d=219$, then it is easy to check that conditions (C1)-(C3) hold. Consequently, all assumptions of Theorem 3.4 hold and so, by Theorem 3.4, (1.6) has at least three positive solutions $x_{1}, x_{2}$, and $x_{3}$ satisfying, for any given $\tau \in(0,3 / 4)$,

$$
\begin{gathered}
\left\|x_{i}\right\| \leq 219 \quad \text { for } i=1,2,3 \\
2<\min \left\{\left|x_{1}(t)\right|: t \in\left[\frac{1}{16}, \frac{15}{16}\right]_{\mathrm{T}}\right\} \\
1<\min \left\{\left|x_{2}(t)\right|: t \in\left[\frac{1}{16}, \frac{15}{16}\right]_{\mathrm{T}}\right\} \quad \text { with } \min \left\{\left|x_{2}(t)\right|: t \in\left[\frac{1}{16}, \frac{15}{16}\right]_{\mathrm{T}}\right\}<2 \\
\min \left\{\left|x_{3}(t)\right|: t \in[0,1]_{\mathrm{T}}\right\}<1 .
\end{gathered}
$$

\section{Acknowledgment}

This work is Supported by Natural Science Foundation of Zhejiang Province (Y607178) and educational department of Zhejiang province (Y200804716).

\section{References}

[1] S. Hilger, "Analysis on measure chains-a unified approach to continuous and discrete calculus," Results in Mathematics, vol. 18, no. 1-2, pp. 18-56, 1990.

[2] M. Bohner and A. Peterson, Dynamic Equations on Time Scales: An Introduction with Applications, Birkhäuser, Boston, Mass, USA, 2001.

[3] V. Lakshmikantham, S. Sivasundaram, and B. Kaymakcalan, Dynamic Systems on Measure Chains, vol. 370 of Mathematics and Its Applications, Kluwer Academic Publishers, Dordrecht, The Netherlands, 1996.

[4] F. M. Atici and G. Sh. Guseinov, "On Green's functions and positive solutions for boundary value problems on time scales," Journal of Computational and Applied Mathematics, vol. 141, no. 1-2, pp. 75-99, 2002. 
[5] R. P. Agarwal and M. Bohner, "Basic calculus on time scales and some of its applications," Results in Mathematics, vol. 35, no. 1-2, pp. 3-22, 1999.

[6] F. M. Atici, G. Sh. Guseinov, and B. Kaymakçalan, “On Lyapunov inequality in stability theory for Hill's equation on time scales," Journal of Inequalities and Applications, vol. 5, no. 6, pp. 603-620, 2000.

[7] D. R. Anderson, "Solutions to second-order three-point problems on time scales," Journal of Difference Equations and Applications, vol. 8, no. 8, pp. 673-688, 2002.

[8] R. P. Agarwal and D. O'Regan, "Nonlinear boundary value problems on time scales," Nonlinear Analysis: Theory, Methods \& Applications, vol. 44, no. 4, pp. 527-535, 2001.

[9] Z. He, "Double positive solutions of three-point boundary value problems for $p$-Laplacian dynamic equations on time scales," Journal of Computational and Applied Mathematics, vol. 182, no. 2, pp. 304-315, 2005.

[10] E. R. Kaufmann, "Positive solutions of a three-point boundary-value problem on a time scale," Electronic Journal of Differential Equations, vol. 2003, no. 82, pp. 1-11, 2003.

[11] R. W. Leggett and L. R. Williams, "Multiple positive fixed points of nonlinear operators on ordered Banach spaces," Indiana University Mathematics Journal, vol. 28, no. 4, pp. 673-688, 1979.

[12] R. I. Avery and J. Henderson, "Two positive fixed points of nonlinear operators on ordered Banach spaces," Communications on Applied Nonlinear Analysis, vol. 8, no. 1, pp. 27-36, 2001.

[13] S. Hong, "Triple positive solutions of three-point boundary value problems for $p$-Laplacian dynamic equations on time scales," Journal of Computational and Applied Mathematics, vol. 206, no. 2, pp. 967-976, 2007.

[14] R. I. Avery and A. C. Peterson, "Three positive fixed points of nonlinear operators on ordered Banach spaces," Computers \& Mathematics with Applications, vol. 42, no. 3-5, pp. 313-322, 2001.

[15] H. Su, Z. Wei, and B. Wang, "The existence of positive solutions for a nonlinear four-point singular boundary value problem with a $p$-Laplacian operator," Nonlinear Analysis: Theory, Methods $\mathcal{E}$ Applications, vol. 66, no. 10, pp. 2204-2217, 2007. 